\title{
sciendo
}

\section{IDENTIFYING BIOMARKERS OF AUTOPHAGY AND APOPTOSIS IN TRANSFECTED NUCLEAR DONOR CELLS AND TRANSGENIC CLONED PIG EMBRYOS*}

\author{
Ju-Young Lee ${ }^{1}$, Sang Hwan Kim² ${ }^{2}$ Jong Taek Yoon ${ }^{1,2,3 \bullet}$ \\ ${ }^{1}$ The Graduate School of Future Convergence Technology, Hankyong National University, Ansung, \\ Gyeonggi-do, 456-749, Korea \\ ${ }^{2}$ Institute of Genetic Engineering, Hankyong National University, Ansung, Gyeonggi-do, \\ 456-749, Korea \\ ${ }^{3}$ Department of Animal Life Science, Hankyong National University, Ansung, Gyeonggi-do, \\ 456-749, Korea \\ •Corresponding author: jtyoon@hknu.ac.kr
}

\begin{abstract}
In this study, we first investigated the effects of 3-methyladenine (3-MA), an autophagy inhibitor, and the inducer - rapamycin (RAPA) on the incidence of programmed cell death (PCD) symptoms during in vitro development of porcine somatic cell nuclear transfer (SCNT)-derived embryos. The expression of autophagy inhibitor mTOR protein was decreased in porcine SCNT blastocysts treated with 3MA. The abundance of the autophagy marker LC3 increased in blastocysts following RAPA treatment. Exposure of porcine SCNT-derived embryos to 3-MA suppressed their developmental abilities to reach the blastocyst stage. No significant difference in the expression pattern of PCD-related proteins was found between non-transfected dermal cell and transfected dermal cell groups. Additionally, the pattern of PCD in SCNT-derived blastocysts generated using SC and TSC was not significantly different, and in terms of porcine SCNT-derived embryo development rates and total blastocyst cell numbers, there was no significant difference between non-transfected cells and transfected cells. In conclusion, regulation of autophagy affected the development of porcine SCNT embryos. Regardless of the type of nuclear donor cells (transfected or non-transfected dermal cells) used for SCNT, there was no difference in the developmental potential and quantitative profiles of autophagy/apoptosis biomarkers between porcine transgenic and non-transgenic cloned embryos. These results led us to conclude that PCD is important for controlling porcine SCNT-derived embryo development, and that transfected dermal cells can be utilized as a source of nuclear donors for the production of transgenic cloned progeny in pigs.
\end{abstract}

Key words: porcine, 3-methyladenine, rapamycin, somatic cell nuclear transfer, autophagy, apoptosis, cloned embryo

\footnotetext{
*This research is supported by the research capacity scholarship of 2015 Hankyong National Uni-
} versity. 
Somatic cell nuclear transfer (SCNT) is a useful technology with application in animal cloning. Since the creation of the first cloned animal, Dolly the sheep, by means of SCNT (Wilmut et al., 1997), cloned specimens have been produced in several other domesticated mammalian species including mice (Tanabe et al., 2017), rabbits (Skrzyszowska et al., 2006), cattle (Luo et al., 2015), goats (Feng et al., 2015), sheep (Zhang et al., 2013), horses (Olivera et al., 2018), and pigs (Kwon et al., 2017). In addition, SCNT has been used to produce transgenic (TG) animals (Kolber-Simonds et al., 2004; Samiec, 2004; Samiec and Skrzyszowska, 2011a; Luo et al., 2015) and cloned endangered species (Samiec, 2005; Gómez and Pope, 2015; Wani et al., 2017). Because of their anatomical and physiological similarity to humans, transgenic pigs have been widely used in biomedical studies, both as models for studying human hereditary diseases and disease resistance (Meurens et al., 2012; Callesen et al., 2017; Staunstrup et al., 2017), and for preclinical/clinical xenotransplantation research (Vajta, 2007; Samiec and Skrzyszowska, 2011 b; Kim et al., 2017; Lee et al., 2017). Although transgenic cloned pigs have been generated by SCNT, disappointingly low efficiency of this assisted reproductive technology (ART) in this livestock species severely limits its extraordinarily broad application spectrum. Recent studies have identified several factors affecting the generation of transgenic or non-transgenic SCNT-derived embryos, fetuses and progeny in pigs, including among others: 1) meiotic, epigenetic and cytoplasmic maturity of nuclear recipient oocytes (Samiec and Skrzyszowska, 2012 a; Wang et al., 2018); 2) the type of nuclear donor cells (Keefer, 2008; Samiec and Skrzyszowska, 2010 a; Li et al., 2013; Samiec et al., 2015); 3) epigenetic reprogrammability of somatic cell nuclei (Lee et al., 2013; Opiela et al., 2017; Jin et al., 2018; Samiec and Skrzyszowska, $2018 \mathrm{a}, \mathrm{b})$; 4) the strategies applied to artificial activation of nuclear-transferred oocytes (Samiec and Skrzyszowska, 2010 b, 2012 b, 2014; Samiec et al., 2012); and 5) the incidence of programmed cell death (PCD) in the in vitro cultured nuclear donor cells and cloned embryos (Samiec and Skrzyszowska, 2013; Samiec et al., 2013 a; Lin et al., 2016; Jin et al., 2017; Zhang et al., 2017). However, the role of PCD pathways (apoptosis and autophagy) in nuclear donor cells and mammalian SCNTderived embryos is still poorly understood (Song et al., 2001; Samiec et al., 2013 b; Shen et al., 2015; Chi et al., 2017; Wang et al., 2017; Agrawal et al., 2018; Zhang et al., 2018).

The availability of healthy donor cells is one of the most important factors for successful SCNT (Mastromonaco et al., 2003). Successful SCNT is only possible using normal diploid cells before they undergo senescence and death (Denning et al., 2001). PCD is important for maintaining homeostasis in multicellular organisms. There are two major types of PCD, defined in large part by the morphological appearance of the dying cell: apoptosis (also known as type I cell death), and autophagy (type II) (Galluzzi et al., 2007). These play the complex relationship between apoptosis and autophagy on mammalian cells (Jia et al., 1997; Jia et al., 2014). Apoptosis and autophagy are essential processes which use DNA fragmentation and degrade organelles to remove unneeded cell components in response to various stresses in order to maintain cellular quality (Xu et al., 2012; Brill et al., 1999). In particular, autophagy plays a role in the regulation of cell survival and death. It has pro-sur- 
vival functions to prevent cellular stress and apoptosis (Tsukada and Ohsumi, 1993; Schwartz et al., 1993).

PCD is related to embryonic development and blastocyst quality, which is important for mammalian embryogenesis (Xu et al., 2012; Brill et al., 1999; Tsukamoto, 2014; Tsukamoto and Yamamoto, 2013). Apoptosis influences the blastocyst quality by regulating cell numbers in embryos, and has been related to early-stage embryonic arrest and fragmentation (Fabian et al., 2005; Yu et al., 2011). Recent studies have shown that the developmental competences of SCNT-derived embryos may be enhanced by treatment of cell cultures with compounds that reduce apoptosis occurrence (e.g., trichostatin A or resveratrol) (Himaki et al., 2011; Ji et al., 2013; Kim et al., 2015; Lee et al., 2015). Autophagy is an essential process in mammalian embryogenesis, and its failure may cause developmental arrest in early embryos (Tsukamoto and Yamamoto, 2013). The pro-survival functions of autophagy are selectively regulated to ensure embryo quality and developmental competence (Tsukamoto, 2014; Lee et al., 2016).

However, the molecular mechanisms underlying the interplay between apoptosis and autophagy in the mammalian embryo are not yet clear. Although apoptosis in porcine embryos has been recently studied, and it has been established that the regulation of autophagy is important for the control of porcine embryo quality and development, research into SCNT has rarely been reported.

To address this lack of knowledge, we investigated the effects of autophagyregulating factors (e.g., 3-methyladenine; 3-MA and rapamycin; RAPA) on the in vitro developmental outcome and molecular quality of porcine cloned embryos, and the incidence of PCD on the molecular quality of transfected or non-transfected nuclear donor cells and the resultant transgenic or non-transgenic SCNT-derived embryos, with the aim of improving the production of high-quality porcine cloned embryos.

\section{Material and methods}

\section{Animals and chemical treatment}

Porcine ear tissues and ovaries were collected at local abattoirs (Pyung-teak, Korea) and transported to the laboratory. All chemicals used in this study were purchased from the Sigma-Aldrich Chemical Company (St. Louis, MO, USA), unless otherwise stated.

3-MA and RAPA were dissolved in PZM-3 medium. SCNT-derived embryos were cultured in the medium supplemented with 3-MA or RAPA and developed from zygote to blastocyst stage (for 7 days). Antibiotic drugs were used at fixed final concentrations (3-MA: $2.5 \mathrm{mM}$ or RAPA: $100 \mathrm{nM}$ ) in accordance with previous studies (Xu et al., 2012; Lee et al., 2011). Antibodies used are listed in Table 1. 
Table 1. List of used antibodies

\begin{tabular}{lccccc|c|c}
\hline \multicolumn{1}{c|}{ Antibody } & Company & Cat\# & Source & IF & ELISA & WB \\
\hline Primary antibodies & & & & & & \\
ATG5 & Abfrontier & LF-PA41338 & rabbit & $1: 100$ & $1: 1000$ & \\
Bcl-2 & Santa Cruz & sc-492 & rabbit & $1: 100$ & $1: 1000$ & \\
Beclin-1 & Abcam & ab231341 & rabbit & $1: 100$ & $1: 1000$ & $1: 1000$ \\
caspase-3 & Abcam & ab4051 & rabbit & $1: 100$ & $1: 1000$ & \\
MAP1LC3A & Abcam & ab168803 & rabbit & $1: 100$ & $1: 1000$ & $1: 1000$ \\
MMP-2 & Abcam & ab78796 & mouse & & $1: 1000$ & \\
MMP-9 & Anaspec & AS-54407 & rabbit & & $1: 1000$ & \\
mTOR & Abfrontier & LF-PA20559 & rabbit & $1: 100$ & $1: 1000$ & \\
SQSTM1 & Santa Cruz & sc-25575 & rabbit & $1: 100$ & $1: 1000$ & \\
B-actin & Santa Cruz & sc-47778 & mouse & & & $1: 1000$ \\
Secondary antibodies & & & & & & \\
mouse IgG-HRP & Santa Cruz & sc-2031 & mouse & & $1: 5000$ & $1: 5000$ \\
rabbit IgG-HRP & Santa Cruz & sc-2054 & rabbit & & $1: 5000$ & $1: 5000$ \\
goat IgG-HRP & Abcam & ab6741 & goat & & $1: 5000$ & \\
Alexa fluor594 rabbit IgG & Life Technologies & A-11012 & rabbit & $1: 500$ & & \\
Alexa fluor488 rabbit IgG & Life Technologies & A-11094 & rabbit & $1: 500$ & & \\
\hline
\end{tabular}

\section{Preparation of donor cells}

Ear skin fibroblast cells were cultured from the ear biopsy of an adult pig. The ear biopsy sample was washed several times with Dulbecco's phosphate-buffered saline (DPBS, Gibco). After washing, the tissue was minced with a surgical blade in DPBS, and subsequently digested with collagenase $(200 \mathrm{U} / \mathrm{mL})$ in Dulbecco's modified Eagle's medium (DMEM, Gibco) for $3 \mathrm{hrs}$ at $38.5^{\circ} \mathrm{C}$. The digested samples were then washed in DMEM by centrifugation at $500 \mathrm{rpm}$ for $5 \mathrm{~min}$ to remove any residual enzyme and seeded into a T-25 tissue culture flask. Seeded cells were then cultured in DMEM supplemented with 1\% antibiotic (Gibco) and 10\% fetal bovine serum (FBS, Gibco) at $38.5^{\circ} \mathrm{C}$ in a humidified atmosphere of $5 \% \mathrm{CO}_{2}$ and $95 \%$ air, until they reached confluence. Subculturing was carried out at intervals of 5-7 days following trypsinization for 5 min using $0.25 \%$ trypsin-EDTA (Gibco). The cells were then stored in freezing medium, consisting of 70\% (v/v) DMEM, 10\% (v/v) DMSO, and $20 \%$ (v/v) FBS, in liquid nitrogen after two passages. Before SCNT, cells were thawed and subsequently cultured in 10\% FBS with DMEM for 3-4 days until $80 \%$ confluent to synchronize most of them into G0/G1. Individual cells were recovered from the monolayer by trypsinization for $1 \mathrm{~min}$ and subsequently used for SCNT.

\section{Transfection of donor cells}

Porcine skin fibroblast cells were transfected using eGFP-encoding lentiviral vectors to prepare cultured cell lines. For transfection of donor cells, the lentiviral vector (lentivirus promoter with $\mathrm{H} 1$; pLV-TH) containing the enhanced Green Fluo- 
rescent Protein (eGFP) gene under control of the elongation factor 1 (EF1) promoter was used (Sandrin et al., 2002). The lentivirus vector was produced following the method described by Tanida et al. (2004) and Zakhartchenko et al. (2001), with minor modifications. Donor skin cells were treated with pLV-TH virus supernatant in the presence of $8 \mu \mathrm{g} / \mathrm{mL}$ polybrene for $9 \mathrm{hrs}$ at a multiplicity of infection (MOI) of 10 at $37^{\circ} \mathrm{C}$ in an incubator with $5 \% \mathrm{CO}_{2}$ in air. Transfected skin cells were sorted by Fluorescent Activated Cell Sorting (FACS), and cells displaying elevated levels of GFP expression were selected (data not shown). The transfected cell line was used for SCNT of donor cells.

\section{Oocyte collection and in vitro maturation (IVM)}

Ovaries were collected at local abattoirs (Pyung-teak, Korea) and transported to the laboratory in sterile physiological saline at $37^{\circ} \mathrm{C}$ within $1 \mathrm{hr}$. Cumulus-oocyte complexes (COCs) were aspirated from superficial ovarian follicles with a diameter of 3 to $6 \mathrm{~mm}$, using an 18-gauge needle attached to a $10-\mathrm{mL}$ disposable. The supernatant was removed, and the sediment was washed three times in washing medium containing Hepes-buffered tissue culture medium 199 (TCM-199), $10 \mu \mathrm{L} / \mathrm{mL}$ antibiotic, and $0.3 \%(\mathrm{w} / \mathrm{v})$ bovine serum albumin (BSA). Subsequently, COCs were observed under a stereomicroscope with only those having 3 or more uniform layers of compact cumulus cells and with homogeneous cytoplasm being selected for further work. The selected COCs were transferred into $500 \mu \mathrm{L}$ of IVM medium, which consisted of TCM-199 supplemented with $10 \%$ (v/v) FBS, $15 \mathrm{ng} / \mathrm{mL}$ epidermal growth factor (EGF), $10 \mathrm{IU} / \mathrm{mL}$ of both pregnant mare serum gonadotropin (PMSG) and human chorionic gonadotropin (hCG), and $30 \mu \mathrm{g} / \mathrm{mL}$ kanamycin for 20-22 hrs. After maturation with hormones, the COCs were cultured in hormone-free IVM medium at $39^{\circ} \mathrm{C}$ for an additional $21-22 \mathrm{hrs}$ in humidified air containing $5 \% \mathrm{CO}_{2}$.

\section{Somatic cell nuclear transfer (SCNT)}

After $40 \mathrm{hrs}$ in IVM, denuded oocytes were stained with $5 \mathrm{mg} / \mathrm{mL}$ of Hoechst 33342 for 5 minutes and observed under an inverted microscope equipped with epifluorescence attachment. An oocyte was held with a holding pipette (Origo), and the zona pellucida (ZP) was partially dissected using a $20-\mu \mathrm{m}$ glass pipette to make a slit near the first polar body (PB). Enucleation was performed by aspirating PB and the adjacent cytoplasm containing the metaphase-II (MII) chromosomes using an aspiration pipette in PZM-3 medium containing $3 \mathrm{mg} / \mathrm{mL} \mathrm{BSA}$, and $7.5 \mathrm{mg} /$ $\mathrm{mL}$ of cytochalasin B (CB). During the injection step, a trypsinized donor cell with a smooth cell surface was transferred into the perivitelline space of an enucleated oocyte in PZM-3 without CB. The resulting SCNT embryo was simultaneously fused and activated with two DC pulses of $1.1 \mathrm{kV} / \mathrm{cm}$ for $30 \mu \mathrm{sec}$ in fusion medium $(0.3$ $\mathrm{M}$ mannitol medium containing $1.0 \mathrm{mM} \mathrm{CaCl} 2 \cdot \mathrm{H}_{2} \mathrm{O}, 0.1 \mathrm{mM} \mathrm{MgCl}_{2} \cdot 6 \mathrm{H}_{2} \mathrm{O}$, and 0.5 $\mathrm{mM}$ HEPES). The reconstructed embryos were then incubated with $7.5 \mu \mathrm{g} / \mathrm{mL} \mathrm{CB}$ in PZM-3 for 4 hrs. After treatment, the embryos were washed with PZM-3 containing $3 \mathrm{mg} / \mathrm{mL}$ BSA, transferred into $50-\mu \mathrm{L}$ micro drops of the same culture media, covered with mineral oil in a polystyrene culture dish, and incubated at $38.5^{\circ} \mathrm{C}$ for 7 days under a $5 \% \mathrm{CO}_{2}$ atmosphere. The day of SCNT was designated as day 1 ; 
cleavage and blastocyst formation were assessed on days 3 and 7 following activation, respectively. To count the total cell numbers in blastocysts, they were collected on day 7, washed in PBS medium, and stained with $5 \mu \mathrm{g} / \mathrm{mL}$ of Hoechst 33342 fluorochrome for $10 \mathrm{~min}$. After a final wash in PBS medium, the blastocysts were mounted on glass slides in a drop of mount, compressed gently with a cover slip, and observed under a fluorescence microscope (Nikon Corp., Tokyo, Japan) at 40× magnification.

\section{Immunofluorescence}

Cells and embryos were fixed in $4 \%$ paraformaldehyde overnight at $4{ }^{\circ} \mathrm{C}$, washed for $30 \mathrm{~min}$ in PBS, and permeabilized with $0.2 \%$ Triton X-100 for $30 \mathrm{~min}$ at room temperature (RT). The samples were blocked at RT for $3 \mathrm{hrs}$ in PBS containing 10\% normal horse serum (NGS) and $0.01 \%$ Triton X-100, and then detected with primary antibodies (diluted 1:100 in blocking buffer) overnight at $4{ }^{\circ} \mathrm{C}$. After washing for 30 min with PBS, the samples were incubated with secondary antibodies (diluted 1:500 in blocking buffer) for $2 \mathrm{hrs}$ at RT and washed with PBS for $30 \mathrm{~min}$. Samples were mounted on slides, stained with DAPI, and observed under a fluorescence microscope (Nikon Corp., Tokyo, Japan) at $200 \times$ and $400 \times$ magnification. Relative fluorescence intensity was analyzed with ImageJ software (National Institutes of Health, Bethesda, MD).

\section{Enzyme-linked immunosorbent assay (ELISA)}

For western blots and ELISA, total protein was extracted from ovarian tissues using Pro-Prep solution (Intron) according to the manufacturer's instructions. Total protein was quantified using a Bradford protein assay kit (Bio-Rad). For quantification of specific protein from the culture medium and cell proteins, samples diluted in assay buffer were used to coat a 96-well ELISA plate overnight at $4{ }^{\circ} \mathrm{C}$. The plate was then washed twice using washing buffer ( $1 \times$ PBS with $2.5 \%$ Triton X-100), and blocked using 1\% BSA blocking solution at RT for $3 \mathrm{hrs}$. Primary antibodies were detected overnight at $4{ }^{\circ} \mathrm{C}$. After washing, immune reactions were detected using secondary antibodies for $2 \mathrm{hrs}$, and substrate solution (BD) was added to initiate the reaction. The reaction was stopped with $1 \mathrm{M} \mathrm{NH}_{2} \mathrm{SO}_{4}$, and absorbency was measured at $450 \mathrm{~nm}$.

\section{Western blot}

Samples containing $20 \mu \mathrm{g}$ of protein were separated by SDS-PAGE (in duplicate) on a $12 \%$ SDS-polyacrylamide gel and transferred to an Immuno-Blot PVDF membrane (Bio-Rad, CA, USA). The membrane was blocked using blocking buffer consisting of $3 \%$ BSA in TBST $(0.1 \%$ Tween $20,50 \mathrm{mM}$ Tris- $\mathrm{HCl}(\mathrm{pH} \mathrm{7.6)}$, $200 \mathrm{mM} \mathrm{NaCl}$ ) for $3 \mathrm{hrs}$ at RT. Following this, the membrane was washed 3 times for 15 min with washing buffer (TBST), followed by overnight incubation at $4^{\circ} \mathrm{C}$ with primary antibodies (diluted 1:1000 in blocking buffer). The membrane was then washed thrice with TBST buffer for $15 \mathrm{~min}$ each, and incubated for 2 hrs with HRPconjugated secondary antibodies (diluted 1:5000 in blocking buffer). Detection was carried out using an ECL detection kit with 5 min incubation in a dark room. The de- 
tection reagent was drained, and the membrane was exposed to a sheet of diagnostic film in a film cassette for 1 to $30 \mathrm{~min}$.

\section{Results}

\section{Effect of 3-MA or RAPA treatment on porcine SCNT embryo development}

SCNT-derived embryos were treated with 3-MA or RAPA during their in vitro culture from one-cell stage to blastocyst stage (Table 2; Figure 1). In the 3-MA-treated group, embryo cleavage activity $(64.7 \pm 3.6 \%)$ and the blastocyst formation rate $(16.6 \pm 5.0 \%)$ were significantly lower than those noticed in other groups. However, in the RAPA-treated group, neither cleavage activity $(70.9 \pm 2.2 \%)$ nor developmental potential to reach the blastocyst stage $(29.3 \pm 3.5 \%)$ differed significantly from the adequate rates observed in the control, i.e., untreated, group $(72.5 \pm 5.2 ; 27.7 \pm 7.1 \%$, respectively; $\mathrm{P} \geq 0.05$ ). This indicated that the in vitro developmental capabilities of porcine cloned embryos are remarkably dependent on the 3-MA-mediated regulation (inhibition/suppression) of autophagy processes.

Table 2. Effect of 3-MA or RAPA in development of SCNT embryos

\begin{tabular}{l|c|c|c}
\hline \multirow{2}{*}{ Type } & \multirow{2}{*}{ No. of embryos cultured } & \multicolumn{2}{|c}{$\%$ of embryos developed to } \\
\cline { 3 - 4 } & & $\geq 2$ cell & total blastocyst \\
\hline CON & 137 & $72.5 \pm 5.2$ & $27.7 \pm 7.1$ \\
$3-M A$ & 133 & $64.7 \pm 3.6$ & $16.6 \pm 5.0$ \\
RAPA & 134 & $70.9 \pm 2.2$ & $29.3 \pm 3.5$ \\
\hline
\end{tabular}

Con: control (untreated) group, 3-MA: 3-methyladenine-treated group, RAPA: rapamycin-treated group.
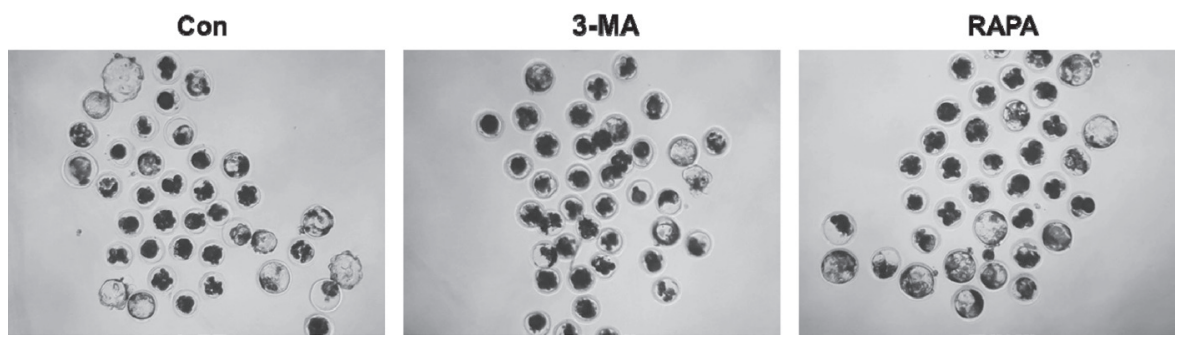

Con: control treatment group, 3-MA: 3-methyadenine treated group, RAPA: rapamycin treated group.

Figure 1. Representative photographs of blastocyst developed in 3-MA or RAPA for the 7 days of IVC

\section{Effect of 3-MA or RAPA treatment on the secretion of MMPs in SCNT em- bryo cultured medium}

We examined the effect of drugs (3-MA or RAPA) on the secretion levels of MMPs in medium-cultured embryos by ELISA (Figure 2). MMP-2 levels were elevated in the 3-MA-treated group, but there was no significant difference between 
the control and RAPA-treated groups. Although the absolute level of MMP-9 secretion was lower than that of MMP-2, MMP-9 secretion patterns between the groups were similar to those found with MMP-2.

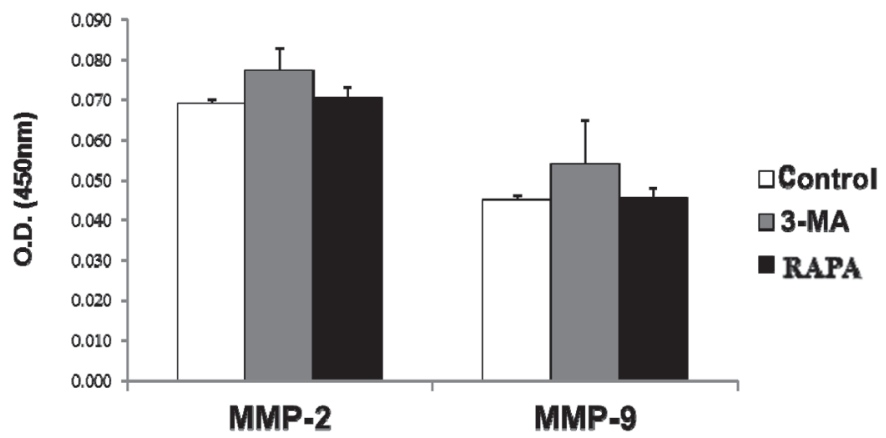

Con: control treatment group, 3-MA: 3-methyadenine treated group, RAPA: rapamycin treated group.

Figure 2. ELISA analysis of secretion MMPs in SCNT culture medium

\section{Effect of 3-MA or RAPA treatment on the expression of autophagy-related proteins in porcine SCNT-derived blastocysts}

Porcine SCNT blastocysts were produced in vitro with 3-MA or RAPA treatment. The relative levels of mTOR (an autophagy inhibitor) and LC3 (an autophagy marker) in blastocysts were measured by immunofluorescence (Figure 3). The relative fluorescence intensity of mTOR was significantly lower in RAPA-treated blastocysts than that noticed in other groups. Blastocysts derived following 3-MA treatment exhibited reduced levels of LC3 $(\mathrm{P}<0.05)$. As compared to the control group, the blastocysts generated after RAPA treatment showed an increased expression pattern of LC3 protein $(\mathrm{P}<0.05)$.

The expression pattern of PCD-related proteins in porcine transfected nuclear donor cells

In order to analyze the expression pattern of apoptosis and autophagy from transfected and non-transfected skin cell lines, expression of target proteins was detected via ELISA and immunofluorescence (Figures 4-7). The results of immunocytobiochemical analysis in both types of nuclear donor cells showed that the expression of anti-apoptotic Bcl-2 protein was considerably higher than that of the apoptosisrelated caspase-3 enzyme. Although such autophagy-regulating proteins as Beclin-1 and mTOR were expressed at a higher level, there was no significant difference in their expression between transfected and non-transfected cell lines $(\mathrm{P} \geq 0.05)$ in contrast to the expression of other autophagy-associated proteins (ATG5, SQSTM1 and MAP1LC3A) that underwent significant enhancement in transfected cell lines as compared to their non-transfected counterparts $(\mathrm{P}<0.05)$. To determine when autophagy actually occurred, conversion of LC3-I to LC3-II, an indicator of autophagy induction, was measured in SC and TSC using western blots. LC3-II levels were expressed, and not significant in both cell lines. 


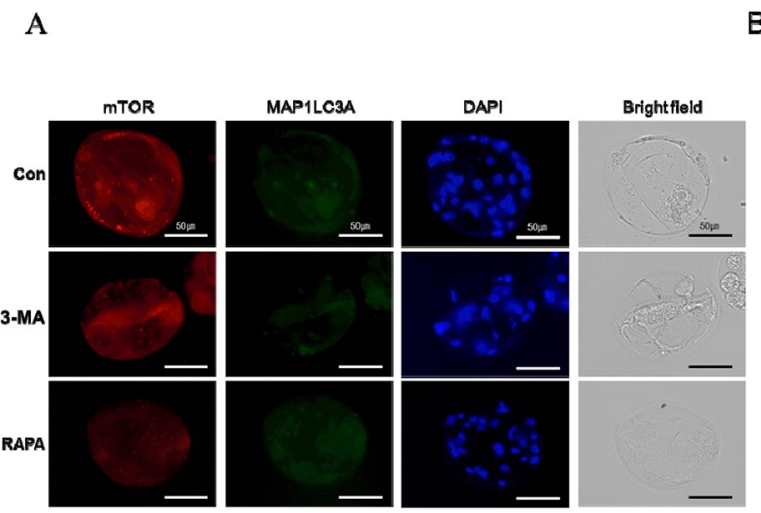

B
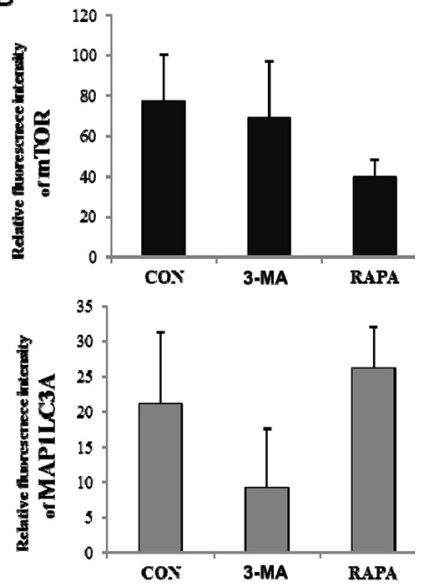

A: Immunofluorescence images of mTOR and MAP1LC3A in cloned embryos not subjected and subjected to 3-MA or RAPA treatment. $(\times 40)$ B: Relative fluorescence level of mTOR and MAP1LC3A in SCNT-derived embryos not undergoing and undergoing exposure to 3-MA or RAPA.

(Con: control/untreated group, 3-MA: 3-methyladenine-treated group, RAPA: rapamycin-treated group).

Figure 3. Effects of 3-MA and RAPA on the autophagy incidence in SCNT-derived blastocysts

A

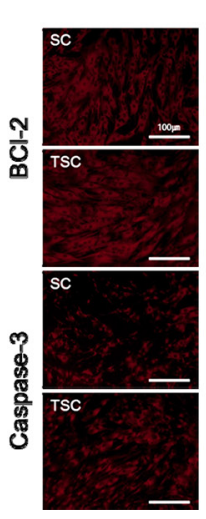

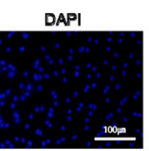
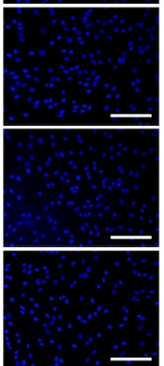
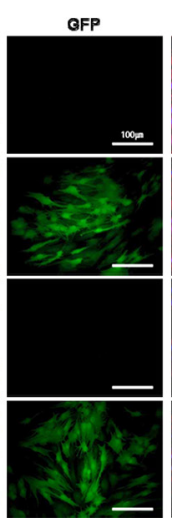

B

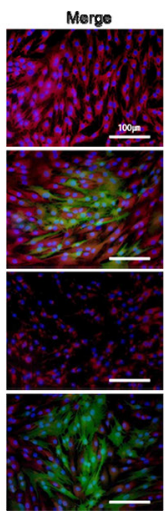

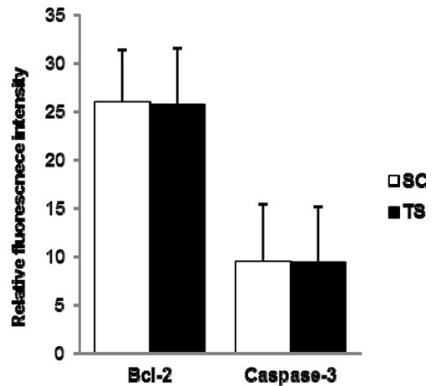

Immunofluorescence images of Bcl-2 and Caspase-3 in somatic cells. $(\times 40)$, SC: skin cell (non-transfection), TSC: transfected skin.

Figure 4. Expression of apoptosis related proteins in somatic cells 
A
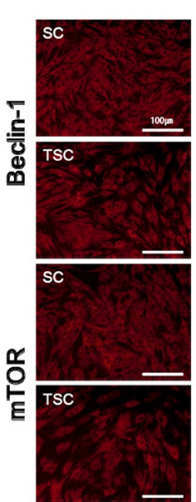
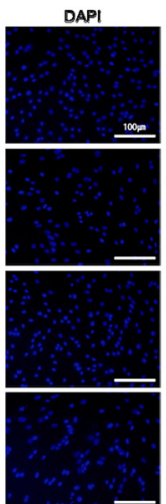
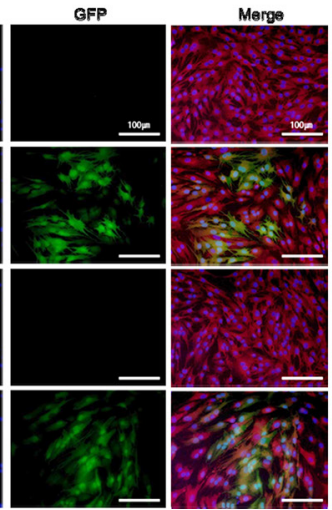

B

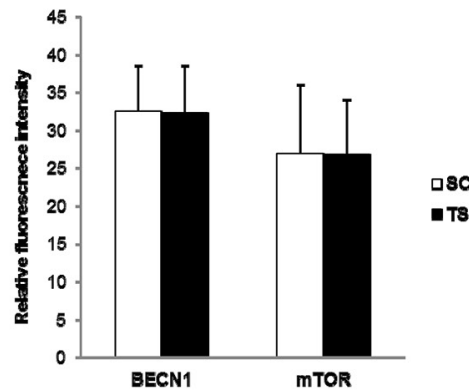

Immunofluorescence images of Beclin- 1 and mTOR in somatic cells. $(\times 40)$, SC: skin cell, TSC: transfected skin cell.

Figure 5. Expression of pro-autophagy related proteins in somatic cells

A

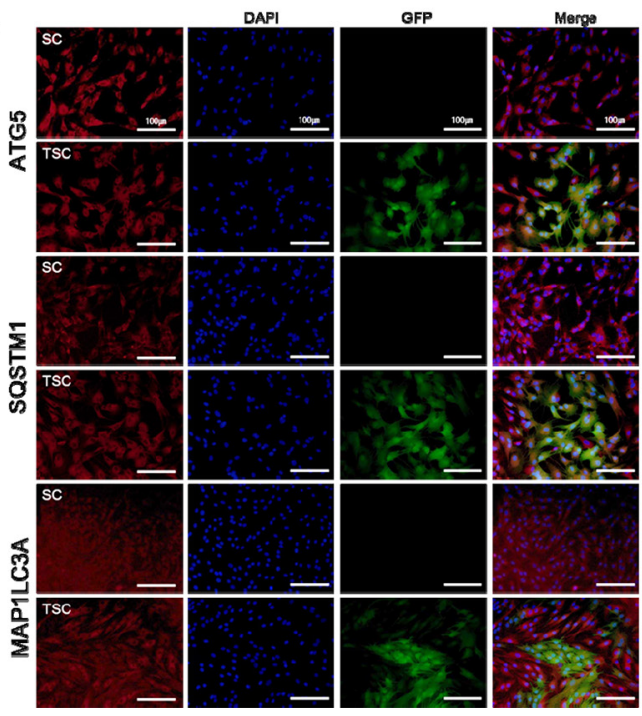

B

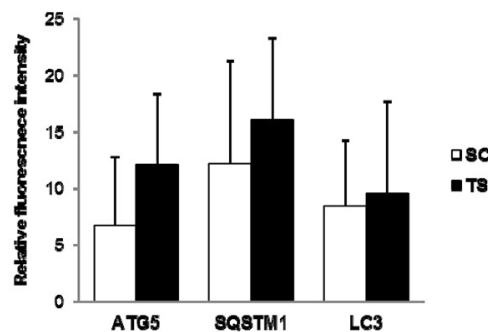

Immunofluorescence images of ATG5 and SQSTM1 and MAP1LC3A in somatic cells. (×40), SC: skin cell, TSC: transfected skin cell.

Figure 6. Expression of autophagy-related protein in somatic cells 
A

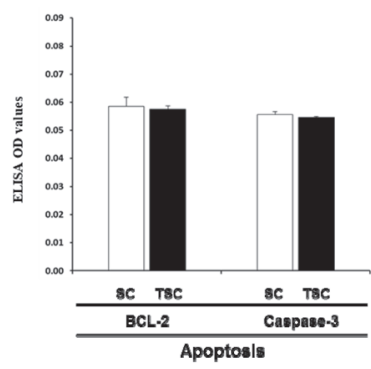

B

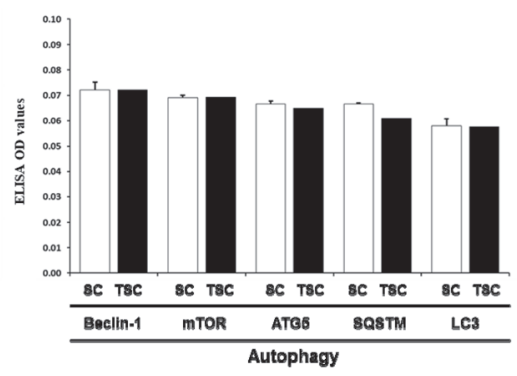

SC TSC

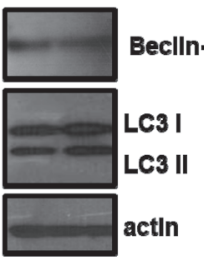

A: ELISA B: western blot, SC: skin cell (non-transfection), TSC: transfected skin cell, Beclin-1: 60kDa, LC3 I and II: 16kDa and 14kDa.

Figure 7. ELISA and western blot of programmed cell death in somatic cells

\section{Effect of nuclear donor cell type on the in vitro developmental competences of cloned pig embryos}

Porcine SCNT embryos were constructed using different donor cell types (SC and TSC). The developmental rate and blastocyst cell numbers are presented in Table 3 . The cleavage rates and blastocyst formation rate did not differ significantly between the two donor cell types $(76.2 \pm 1.2 \%, 32.6 \pm 2.2 \%$ vs $76.4 \pm 0.9 \%, 33.1 \pm 1.8 \%$ : for SC vs TSC), and there was no significant difference among groups in terms of total cell numbers in blastocysts $(30.86 \pm 2.76 \%$ vs $30.43 \pm 2.50 \%)$.

Table 3. Effect of donor cell in development rate of SCNT embryos

\begin{tabular}{l|c|c|c|c}
\hline \multirow{2}{*}{ Cell type } & \multirow{2}{*}{$\begin{array}{c}\text { No. of } \\
\text { embryos cultured }\end{array}$} & \multicolumn{2}{|c|}{$\%$ of embryos developed to } & \multirow{2}{*}{ No. of cells in blastocyst (n) } \\
\cline { 3 - 4 } & 126 & $\geq 2$ cell & total blastocyst & \\
\hline SC & 149 & $76.2 \pm 1.2$ & $32.6 \pm 2.2$ & $30.86 \pm 2.76$ \\
TSC & $76.4 \pm 0.9$ & $33.1 \pm 1.8$ & $30.43 \pm 2.50$ \\
\hline
\end{tabular}

Expression patterns of PCD-related proteins in the blastocysts developed from porcine SCNT-derived embryos reconstructed with transfected dermal cells

To examine the expression of genes related to embryonic development and PCD, we evaluated the expression levels of Bcl-2, Caspase-3, mTOR, Beclin-1, ATG5, and MAP1LC3A in blastocysts from each group using immunofluorescence (Figures 8-9). The expression pattern of PCD in blastocysts from SC and TSC embryos did not differ significantly. Moreover, results from cloned embryos indicated that PCD expression level patterns were similar to those seen in donor cells. However, in contrast with the donor cell experiment, MAP1LC3A levels in SCNT blastocysts were lower than those of other autophagy-related proteins. 
A
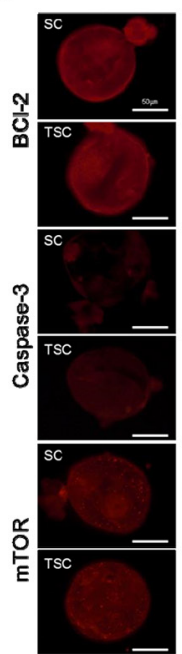

DAPI

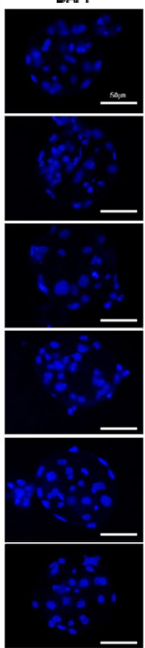

afp

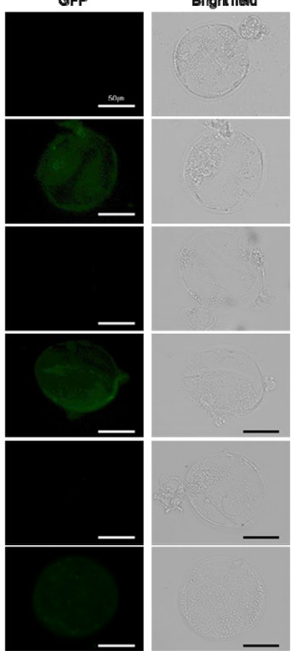

B

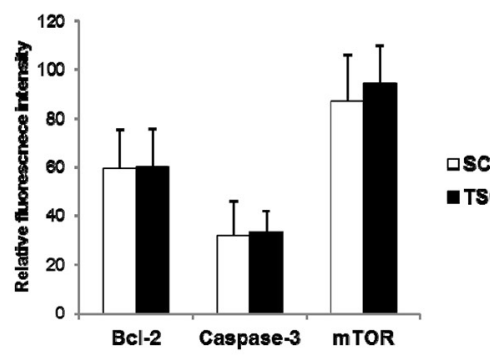

Immunofluorescence images of Bcl-2 and Caspase- 3 and mTOR in SCNT blastocyst $(\times 40)$, SC: skin cell (non-transfection), TSC: transfected skin cell.

Figure 8. Expression of apoptosis related protein in SCNT blastocyst

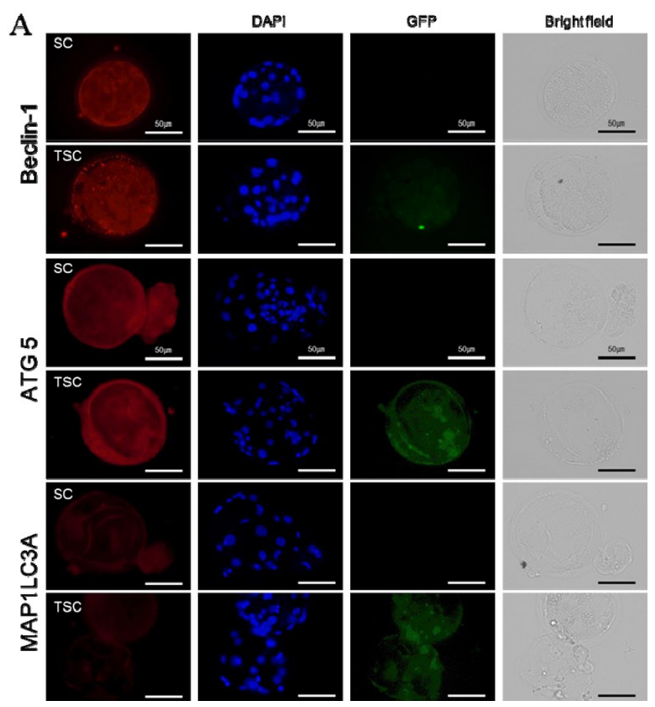

B

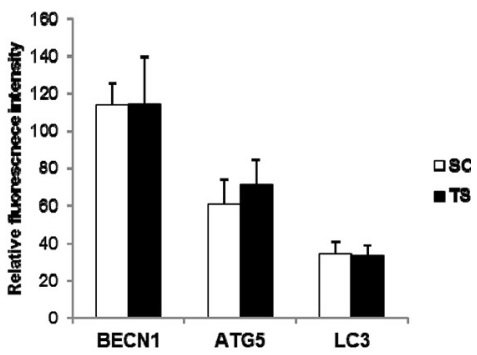

A: Immunofluorescence images of Beclin-1 and ATG5 and MAP1LC3A in SCNT blastocyst $(\times 40)$, B: autophagy related proteins, SC: skin cell (non-transfection), TSC: transfected skin cell.

Figure 9. Expression of autophagy related protein in SCNT blastocyst 


\section{Discussion}

SCNT has enormous potential for xenotransplantation to humans by using TG pigs as tissue and organ donors (Samiec, 2004; Samiec and Skrzyszowska, 2011 b; Kim et al., 2017; Kwon et al., 2017; Lee et al., 2017). However, to further advance the production of genetically modified cloned pigs, more research focused on the efficient generation of porcine SCNT-derived embryos and offspring is required. Various factors contribute to SCNT's low efficiency, especially donor cell type, donor cell transfection, culture conditions, apoptosis, and autophagy (PCD). PCD plays a critical role in pre-implantation development of porcine embryos produced in vitro for cellular maintenance (Tsukamoto, 2014). Apoptosis has been related to embryonic arrest and fragmentation in early-stage embryos (Yu et al., 2011), and autophagy is an essential process for pre-implantation embryo development in mammals (Tsukamoto and Yamamoto, 2013).

In this study, we first examined the effects of the autophagy inhibitor 3-MA and inducer RAPA on PCD expression on porcine SCNT-derived embryo development. The expression of mTOR protein was decreased in porcine SCNT blastocysts treated with 3-MA. In contrast, the abundance of the autophagy marker LC3 increased in blastocysts following RAPA treatment. These results indicate that treatment with 3-MA or RAPA alters the expression of autophagy-related genes in porcine SCNT embryos, which is consistent with the regulation of autophagy by these drugs in other cells (Pan et al., 2009). Furthermore, this regulation of autophagy influences porcine embryo development.

Treatment of SCNT embryos with 3-MA had the strongest effect on diminishing blastocyst development rate. These results are consistent with those of recent studies involving porcine parthenogenesis and in vitro fertilization of embryos (Xu et al., 2012; Lee et al., 2016). Autophagy plays a critical role in early embryogenesis, where it is involved in the degradation of maternal proteins and synthesis of new proteins. The failure of these processes causes developmental arrest and low blastocyst development rates (Tsukamoto and Yamamoto, 2013). Therefore, our results support the conclusion that the inhibition of autophagy negatively affects porcine SCNTderived blastocyst development.

The blastocyst formation rate within the RAPA-treated group was higher than that in the control group, but not significantly different from that in the 3-MA-treated group. In previous investigations, whereas rapamycin treatment did not affect significantly the competences of porcine parthenogenetic embryos to complete their development to blastocyst stage ( $\mathrm{Xu}$ et al., 2012), such exposure of early-stage bovine in vitro-fertilized embryos led to enhancement of the blastocyst yields (Song et al., 2012). Rapamycin has a protective effect on cell development and survival during starvation, but does not affect cellular maintenance under normal culture conditions (Patricia et al., 2005). Under normal conditions, basal levels of autophagy induce a pro-survival mechanism, whereas prolonged or excessive induction of autophagy stress can promote cell death (Knight and Shokat, 2007). Rapamycin treatment may influence embryo development, either through beneficial or detrimental effects (Song et al., 2012). We found that autophagy induced by rapamycin treatment may provi- 
sionally have positive effects on the rate of porcine SCNT-derived blastocyst development. An increased level of MMP secretion was found in the 3-MA-treated group, which exhibited the lowest development rate of SCNT-derived embryos. MMPs play an important role in tissue remodeling of cells through their interaction with ECM (Deryugina and Quigley, 2006; Malemud et al., 2006). The activity of MMPs was increased during inhibition of cell development and induction of apoptosis (Galoian et al., 2012; Lee et al., 2010; Page-McCaw et al., 2007). MMPs may therefore be associated with the development of porcine SCNT-derived embryos and the modulation of autophagy.

Transfection of donor cells may affect the development of porcine TG-SCNT-derived embryos. High-quality donor cells are needed both to get high-quality embryos and to improve the efficiency of TG-SCNT (Wongsrikeao et al., 2007). Transfection of donor cells requires a long period of in vitro culture (IVC) and selection of marker genes, and it has been suggested that this extended culture time may induce transfection stress, thereby decreasing TG-SCNT efficiency (Zakhartchenko et al., 2001). The quality of embryos reconstructed with gene-transfected donor cells is an important factor in the production of TG pigs using SCNT (Brink et al., 2000; Brophy et al., 2003; Iguma et al., 2005).

We next examined the expression pattern of PCD-related proteins in transfected cutaneous cells used as nuclear donors for generation of porcine SCNT-derived embryos. There was no significant difference between expression patterns found in skin cell (SC) and transfected skin cell (TSC) groups for any of the PCD-related proteins analyzed (Bcl-2, Caspase-3, mTOR, Beclin-1, ATG5, SQSTM1 and MAP1LC3A). These results suggest that lentivirus-mediated transfection of nuclear donor dermal cells does not influence post-transfection intracellular stress that has been measured by the relative abundance of markers peculiar to apoptosis and autophagy, as compared to their expression in non-transfected dermal cells. Anti-apoptotic Bcl-2 was highly expressed in both cell types, whereas the expression of apoptosis marker caspase-3 was comparatively low in both groups. Proteins related to autophagy occurrence were generally expressed at a higher level. Bcl-2 and mTOR, which are crucial to cell survival, were highly expressed. Therefore, it was concluded that transfected cells do not display apoptosis under normal growth conditions. However, LC3 and Beclin-1-related keys of autophagy were highly expressed as mTOR and LC3-II, indicating that autophagy activation was increased in the results of western blot, which was exhibited in both cell survival and autophagy avoiding apoptotic event. Previous studies have claimed that embryos must be reconstructed with G0/G1 stage donor cells when oocytes with higher levels of maturation-promoting factors are used as recipients in SCNT (Wilmut et al., 1997; Cibelli et al., 1998; Kasinathan et al., 2001). Although several methods have been used for artificial synchronization of mitotic cycle of nuclear donor cells at the G1/G0 or G0/G1 phases, their contact inhibition under total confluence conditions seems to be the most frequently used method for the purposes of SCNT in pigs (Miyamoto et al., 2007; Verma et al., 2015; Keefer, 2015; Samiec and Skrzyszowska, 2012 b; Samiec et al., 2013 a, b). Autophagy promotes cellular survival in response to the external environmental stressors (e.g., serum starvation) (Zhang et al., 2018). Consequently, we considered that the activation 
of autophagy in donor cells occurred in response to the synchronization of donor cells at the $\mathrm{G} 1 / \mathrm{G} 0$ phases.

We examined the production of SCNT-derived embryos using transfected skin cells and analyzed embryo development rates and the expression of PCD in SCNTderived blastocysts. There was no significant difference in the in vitro developmental rates or blastocyst yields between porcine SCNT-derived embryos created using transfected cutaneous cells and their counterparts created using non-transfected cutaneous cells. Moreover, the expression pattern of PCD-related proteins in SCNTderived blastocysts was similar to that seen in donor cells, with the sole exception of LC3. This was consistent with previous research showing that LC3 was expressed at a low level during the blastocyst stage, when compared to the early-stage porcine parthenogenetic embryos (Xu et al., 2012). Recent findings suggest that Beclin-1 acts as a crucial regulator of both autophagy and apoptosis (Nikoletopoulou et al., 2013). Disruption of Beclin-1 induces early embryonic lethality and widespread apoptotic cell death in mouse embryos (Wakayama et al., 1998; Yue et al., 2003). Silencing of autophagy-related genes, similarly, leads to developmental disorders and defective embryonic development (Lee et al., 2016). Therefore, we suggest that autophagy plays a critical and selectively pro-survival role in porcine TG-SCNTderived embryos, but that further study is required in order to elucidate the specific molecular mechanism underlying PCD in porcine SCNT-derived cells.

Summing up, the results of our study show that transfected porcine dermal cells did not differ significantly from non-transfected dermal cells with respect to the expression of PCD-associated proteins. Similarly, there was no significant difference in the ex vivo developmental capacity of nuclear-transferred embryos. Furthermore, regulation of autophagy processes by extrinsic agents (3-MA or RAPA) affected not only developmental potential of SCNT-derived pig embryos, but also their molecular quality that was assessed by determining the quantitative profiles of autophagy-related proteins. However, regardless of the type of nuclear donor cells (transfected or non-transfected cutaneous cells) used for SCNT procedure, there was no difference in the blastocyst formation rates and the expression patterns of apoptosis/autophagy biomarkers between porcine transgenic and non-transgenic cloned embryos. Our results support the conclusions that PCD is important for controlling the in vitro development of porcine cloned embryos and that transfected cutaneous cells can be utilized as nuclear donors for the production of transgenic SCNT-derived offspring in pigs.

\section{References}

Agrawal H., Selokar N.L., Saini M., Singh M.K., Chauhan M.S., Palta P., Sing l a S.K., M a n ik R.S. (2018). m-carboxycinnamic acid bishydroxamide improves developmental competence, reduces apoptosis and alters epigenetic status and gene expression pattern in cloned buffalo (Bubalus bubalis) embryos. Reprod. Domest. Anim., 53: 986-996.

B o y a P., González-Polo R.A., Cas ares N., P erfettini J.L., Dessen P., La rochet te N., Métivier D., Meley D., Souquere S., Yoshimori T., Pierron G., Codog n o P., K r o e m e r G. (2005). Inhibition of macroautophagy triggers apoptosis. Mol. Cell Biol., 25: $1025-1040$. 
B rill A., Tor ch in sky A., C a r p H., To d e r V. (1999). The role of apoptosis in normal and abnormal embryonic development. J. Assist. Reprod. Genet., 16: 512-519.

B ri in k M.F., B i s h o p M.D., P i e p e r F.R. (2000). Developing efficient strategies for the generation of transgenic cattle which produce biopharmaceuticals in milk. Theriogenology, 53: 139-148.

Brophy B., Smolenski G., Wheeler T., Wells D., L'Huillier P., Laible G. (2003). Cloned transgenic cattle produce milk with higher levels of beta-casein and kappa-casein. Nat. Biotechnol., 21: 157-162.

Calle sen M.M., Árnadóttir S.S., Lyskjaer I., Ørnt oft M.W., Høyer S., Dagnaes - Hansen F., Liu Y., Li R., Callesen H., Rasmussen M.H., B erthelsen M.F., Thom se n M.K., S chwe iger P.J., J en sen K.B., L a urberg S., Ørntoft T.F., Elver$1 \varnothing \mathrm{v}-\mathrm{J}$ a k o b s e n J.E., A n d e r s e n C.L. (2017). A genetically inducible porcine model of intestinal cancer. Mol. Oncol., 11: 1616-1629.

C a m p b e 11 K.H., M c W h i r J., R it c h i e W.A., W i 1 m u t I. (1996). Sheep cloned by nuclear transfer from a cultured cell line. Nature, 380: 64-66.

Chi D., Zeng Y., X u M., S i L., Qu X., Li u H., L i J. (2017). LC3-dependent autophagy in pig 2-cell cloned embryos could influence the degradation of maternal mRNA and the regulation of epigenetic modification. Cell. Reprogram., 19: 354-362.

Cibelli J.B., Stice S.L., Golueke P.J., Kane J.J., Jerry J., Blackwell C., Ponce de L e ó n F.A., R o b 1 J.M. (1998). Cloned transgenic calves produced from nonquiescent fetal fibroblasts. Science, 280: 1256-1258.

Denning C., Dickinson P., Burl S., Wylie D., Fletcher J., Clark A.J. (2001). Gene targeting in primary fetal fibroblasts from sheep and pig. Clon. Stem Cells, 3: 221-231.

D e ry ug in a E.I., Q u ig ley J.P. (2006). Matrix metalloproteinases and tumor metastasis. Cancer Metastasis Rev., 25: 9-34.

Fabian D., Koppel J., Maddox-Hyttel P. (2005). Apoptotic processes during mammalian preimplantation development. Theriogenology, 64: 221-231.

Feng X., Cao S., Wang H., Meng C., Li J., Jiang J., Q i an Y., S u L., He Q., Zhang Q. (2015). Production of transgenic dairy goat expressing human $\alpha$-lactalbumin by somatic cell nuclear transfer. Transgenic Res., 24: 73-85.

Galluzzi L., Maiuri M.C., Vitale I., Zischka H., Castedo M., Zitvogel L., Kroe mer G. (2007). Cell death modalities: classification and pathophysiological implications. Cell Death Differ., 14: 1237-1243.

Galoi a n K., Temple H.T., Galoy an A. (2012). mTORC1 inhibition and ECM-cell adhesionindependent drug resistance via PI3K-AKT and PI3K-RAS-MAPK feedback loops. Tumour Biol., 33: 885-890.

Gó me z M.C., P ope C.E. (2015). Cloning endangered felids by interspecies somatic cell nuclear transfer. Methods Mol. Biol., 1330: 133-152.

H i m a k i T., Yo k o m in e T.A., S a to M., Tak a o S., Mi y o shi K., Yosh ida M. (2011). Effects of trichostatin A on in vitro development and transgene function in somatic cell nuclear transfer embryos derived from transgenic Clawn miniature pig cells. Anim Sci. J., 81: 558-563.

I g u m a L.T., L i s a u k a s S.F., M e lo E.O., Fran co M.M., P iv a to I., Vi an na G.R., S ou s a R.V., D o de M.A., A ra gã o F.J., R e ch E.L., Rumpf R. (2005). Development of bovine embryos reconstructed by nuclear transfer of transfected and non-transfected adult fibroblast cells. Genet. Mol. Res., 4: 55-66.

Ji Q., Zhu K., Liu Z., Song Z., Huang Y., Zhao H., Chen Y., He Z., Mo D., Cong P. (2013). Improvement of porcine cloning efficiency by trichostain A through early-stage induction of embryo apoptosis. Theriogenology, 79: 815-823.

J i a L., D our m a sh kin R.R., A 11 e n P.D., G ra y A.B., N ew 1 and A.C., Ke 1 s e y S.M. (1997). Inhibition of autophagy abrogates tumour necrosis factor alpha induced apoptosis in human T-lymphoblastic leukaemic cells. Br. J. Haematol., 98: 673-685.

Jia L., Dourmashkin R.R., Allen P.D., Gray A.B., Newland A.C., Kelsey S.M. (2014). Self-consumption: the interplay of autophagy and apoptosis. Nat. Rev. Mol. Cell Biol., 15: $81-94$.

Jin L., Guo Q., Zhu H.Y., Xing X.X., Zhang G.L., Xuan M.F., Luo Q.R., Luo Z.B., W a n g J.X., Y in X.J., K a n g J.D. (2017). Quisinostat treatment improves histone acetylation and 
developmental competence of porcine somatic cell nuclear transfer embryos. Mol. Reprod. Dev., 84: 340-346.

Jin L., Guo Q., Zhang G.L., Xing X.X., Xuan M.F., Luo Q.R., Luo Z.B., Wang J.X., Y in X.J., Kang J.D. (2018). The histone deacetylase inhibitor, CI994, improves nuclear reprogramming and in vitro developmental potential of cloned pig embryos. Cell. Reprogram., 20: $205-213$.

K a s in a than P., Knott J.G., M or e ir a P.N., B urns i d e A.S., J e r r y D.J., R obl J.M. (2001). Effect of fibroblast donor cell age and cell cycle on development of bovine nuclear transfer embryos in vitro. Biol. Reprod., 64: 1487-1493.

K e e fe r C.L. (2008). Lessons learned from nuclear transfer (cloning). Theriogenology, 69: $48-54$.

Ke efer C.L. (2015). Artificial cloning of domestic animals. Proc. Natl. Acad. Sci. USA, 112: 8874-8878.

K i m G.A., L e e E.M., J in J.X., L e e S., Taw e e cha i p a is a n kul A., H wang J.I., A la m Z., A h n C., L e e B.C. (2017). Generation of CMAHKO/GTKO/shTNFRI-Fc/HO-1 quadruple gene modified pigs. Transgenic Res., 26: 435-445.

K i m S.H., Z h a o M.H., L i ang S., C u i X.S., K i m N.H. (2015). Inhibition of cathepsin B activity reduces apoptosis by preventing cytochrome c release from mitochondria in porcine parthenotes. J. Reprod. Dev., 61: 261-268.

Kn ight Z.A., Sh o k a t K.M. (2007). Chemically targeting the PI3K family. Biochem. Soc. Trans., 35: 245-249.

Kolber-Simonds D., Lai L., Watt S.R., Denaro M., Arn S. (2004). Production of alpha-1,3-galactosyltransferase null pigs by means of nuclear transfer with fibroblasts bearing loss of heterozygosity mutations. Proc. Natl. Acad. Sci. USA, 101: 7335-7340.

K w o n D.J., K im D.H., Hwang I.S., K i m D.E., K i m H.J., Kim J.S., Le e K., Im G.S., L e e J.W., Hwang S. (2017). Generation of $\alpha$-1,3-galactosyltransferase knocked-out transgenic cloned pigs with knocked-in five human genes. Transgenic Res., 26: 153-163.

L e e H.R., Gupta M.K., Kim D.H., Hwang J.H., Kw on B., L e e H.T. (2016). Poly(ADP-ribosyl)ation is involved in pro-survival autophagy in porcine blastocysts. Mol. Reprod. Dev., 83: 37-49.

L e e P.S., Ts ang S.W., M o s e s M.A., Traye s-G ib s on Z., H s i a o L.L., J e n s e n R., S quil lace R., Kw iatkowski D.J. (2010). Rapamycin-insensitive up-regulation of MMP2 and other genes in tuberous sclerosis complex 2-deficient lymphangioleiomyomatosis-like cells. Am. J. Respir. Cell Mol. Biol., 42: 227-234.

Lee S., J in J.X., K ho irinaya C., K im G.A., Lee B.C. (2015). Lanosterol influences cytoplasmic maturation of pig oocytes in vitro and improves preimplantation development of cloned embryos. Theriogenology, 61: 261-268.

L e e S.C., L e e H., O h K.B., Hw a ng I.S., Yang H., P a r k M.R., O c k S.A., W o o J.S., I m G.S., Hwang S. (2017). Production and breeding of transgenic cloned pigs expressing human CD73. Dev. Reprod., 21: 157-165.

L e e S.E., Hwang K.C., S u n S.C., X u Y.N., K i m N.H. (2011). Modulation of autophagy influences development and apoptosis in mouse embryos developing in vitro. Mol. Reprod. Dev., 78 : 498-509.

L e e S.H., Xu Y.N., H e o Y.T., Cu i X.S., K i m N.H. (2013). Effects of trichostatin A and 5-aza2'deoxycytidine on nuclear reprogramming in pig cloned embryos. Reprod. Dev. Biol., 37: 269-279.

L i Z., H e X., Ch en L., Sh i J., Z h o u R., X u W., L i u D., W u Z. (2013). Bone marrow mesenchymal stem cells are an attractive donor cell type for production of cloned pigs as well as genetically modified cloned pigs by somatic cell nuclear transfer. Cell. Reprogram., 15: 459-470.

L in T., L e e J.E., O q a n i R.K., K i m S.Y., Cho E.S., J e ong Y.D., B a e k J.J., J in D.I. (2016). Tauroursodeoxycholic acid improves pre-implantation development of porcine SCNT embryo by endoplasmic reticulum stress inhibition. Reprod. Biol., 16: 269-278.

Luo Y., Wang Y., Liu J., Lan H., Shao M., Yu Y., Quan F., Zhang Y. (2015). Production of transgenic cattle highly expressing human serum albumin in milk by phiC31 integrase-mediated gene delivery. Transgenic Res., 24: 875-883.

M a le mud C.J. (2006). Matrix metalloproteinases (MMPs) in health and disease: an overview. Front Biosci., 11: 1696-1701. 
Mastromon a co G.F., P errault S.D., B et t s D.H., K ing W.A. (2003). Role of chromosome stability and telomere length in the production of viable cell lines for somatic cell nuclear transfer. BMC Dev. Biol., 6: 41.

M euren s F., S u m m erfie ld A., N a u w y n ck H., S a if L., Gerd t s V. (2012). The pig: a model for human infectious diseases. Trends Microbiol., 30: 50-57.

Mi y a m o to K., Ho s h in o Y., M in a m i N., Yam a d a M., I m a i H. (2007). Effects of synchronization of donor cell cycle on embryonic development and DNA synthesis in porcine nuclear transfer embryos. J. Reprod. Dev., 53: 237-246.

Nikole top oulou V., Markaki M., Palikaras K., Tavernarakis N. (2013). Crosstalk between apoptosis, necrosis and autophagy. Biochim. Biophys. Acta, 1833: 3448-3459.

Olivera R., Moro L.N., Jordan R., Pallarols N., Guglielminetti A., Luzzani C., Miriuka S.G., Vich era G. (2018). Bone marrow mesenchymal stem cells as nuclear donors improve viability and health of cloned horses. Stem Cells Cloning, 11: 13-22.

O p i e l a J., S a m i e c M., R o m a n e k J. (2017). In vitro development and cytological quality of inter-species (porcine $\rightarrow$ bovine) cloned embryos are affected by trichostatin A-dependent epigenomic modulation of adult mesenchymal stem cells. Theriogenology, 97: 27-33.

P a g e - M c C a w A., Ew a ld A.J., W e r b Z. (2007). Matrix metalloproteinases and the regulation of tissue remodelling. Nat. Rev. Mol. Cell Biol., 8: 221-233.

Pan T., Rawal P., Wu Y., Xie W., Jankovic J., Le W. (2009). Rapamycin protects against rotenone-induced apoptosis through autophagy induction. Neuroscience, 164: 541-551.

S a m i e c M. (2004). Development of pig cloning studies: past, present and future. J. Anim. Feed Sci., 13: $211-238$.

$\mathrm{S}$ a $\mathrm{m}$ i e c M. (2005). The effect of mitochondrial genome on architectural remodeling and epigenetic reprogramming of donor cell nuclei in mammalian nuclear transfer-derived embryos. J. Anim. Feed Sci., 14: 393-422.

S a mi e c M., Skrzyszows ka M. (2010 a). Preimplantation developmental capability of cloned pig embryos derived from different types of nuclear donor somatic cells. Ann. Anim. Sci., 10: 385-398.

S a m i e c M., S krzy s z o w s k a M. (2010 b). The use of different methods of oocyte activation for generation of porcine fibroblast cell nuclear-transferred embryos. Ann. Anim. Sci., 10: 399-411.

S a mi e c M., Skrzys z ow s k a M. (2011 a). Transgenic mammalian species, generated by somatic cell cloning, in biomedicine, biopharmaceutical industry and human nutrition/dietetics - recent achievements. Pol. J. Vet. Sci., 14: 317-328.

S a m i e c M., Skrzy s z ow s k a M. (2011 b). The possibilities of practical application of transgenic mammalian species generated by somatic cell cloning in pharmacology, veterinary medicine and xenotransplantology. Pol. J. Vet. Sci., 14: 329-340.

S a m i e c M., S k r z y s z ow s k a M. (2012 a). High developmental capability of porcine cloned embryos following trichostatin A-dependent epigenomic transformation during in vitro maturation of oocytes pre-exposed to R-roscovitine. Anim. Sci. Pap. Rep., 30: 383-393.

S a mi e c M., Skrzyszowska M. (2012 b). Roscovitine is a novel agent that can be used for the activation of porcine oocytes reconstructed with adult cutaneous or fetal fibroblast cell nuclei. Theriogenology, 78: 1855-1867.

S a m i e c M., S kr z y s z o w s k a M. (2013). Assessment of in vitro developmental capacity of porcine nuclear-transferred embryos reconstituted with cumulus oophorus cells undergoing vital diagnostics for apoptosis detection. Ann. Anim. Sci., 13: 513-529.

S a m i e c M., S k r z y s z o w s k a M. (2014). Biological transcomplementary activation as a novel and effective strategy applied to the generation of porcine somatic cell cloned embryos. Reprod. Biol., 14: $128-139$.

S a m i e c M., S k r z y s z o w s k a M. (2018 a). Intrinsic and extrinsic molecular determinants or modulators for epigenetic remodeling and reprogramming of somatic cell-derived genome in mammalian nuclear-transferred oocytes and resultant embryos. Pol. J. Vet. Sci., 21: 217-227.

S a mi e c M., Skrzyszowska M. (2018 b). Can reprogramming of overall epigenetic memory and specific parental genomic imprinting memory within donor cell-inherited nuclear genome be a major hindrance for the somatic cell cloning of mammals? - a review. Ann. Anim. Sci., 18: 623-638. 
S a mi e c M., Skrzyszow ska M., Li piński D. (2012). Pseudophysiological transcomplementary activation of reconstructed oocytes as a highly efficient method used for producing nucleartransferred pig embryos originating from transgenic foetal fibroblast cells. Pol. J. Vet. Sci., 15: 509-516.

S a mi e c M., Skrzyszows ka M., O piela J. (2013 a). Creation of cloned pig embryos using contact-inhibited or serum-starved fibroblast cells analysed intra vitam for apoptosis occurrence. Ann. Anim. Sci., 13: 275-293.

S a miec M., Skrzyszowska M., B ochenek M. (2013 b). In vitro development of porcine nuclear-transferred embryos derived from fibroblast cells analysed cytometrically for apoptosis incidence and accuracy of cell cycle synchronization at the G0/G1 stages. Ann. Anim. Sci., 13: $735-752$.

S a m i e c M., O p i e l a J., L i p ińs ki D., R o ma nek J. (2015). Trichostatin A-mediated epigenetic transformation of adult bone marrow-derived mesenchymal stem cells biases the in vitro developmental capability, quality, and pluripotency extent of porcine cloned embryos. Biomed. Res. Int., 2015: 814686.

S andrin V., B o s on B., S a 1 mon P., Gay W., N ègre D., L e Grand R., Trono D., Cos s e t F.L. (2002). Lentiviral vectors pseudotyped with a modified RD114 envelope glycoprotein show increased stability in sera and augmented transduction of primary lymphocytes and CD34+ cells derived from human and nonhuman primates. Blood, 100: 823-832.

S chwartz L.M., S mith S.W., Jones M.E., Os borne B.A. (1993). Do all programmed cell deaths occur via apoptosis? Proc. Natl. Acad Sci. USA, 90: 980-984.

Shen X., Zhang N., Wang Z., B a i G., Zheng Z., Gu Y., Wu Y., Liu H., Zhou D., Lei L. (2015). Induction of autophagy improves embryo viability in cloned mouse embryos. Sci. Rep., 5: 17829.

Skrzyszowska M., S morąg Z., Słomski R., Kątska-Ks iążkiewicz L., Kalak R., Michalak E., Wielgus K., Lehmann J., Lipiński D., Szalata M., Pławski A., S a mi e c M., J u r a J., G a j d a B., Ry ń s ka B., P i eń k o w s k i M. (2006). Generation of transgenic rabbits by the novel technique of chimeric somatic cell cloning. Biol. Reprod., 74: 1114-1120.

S ong B.S., Kim J.S., Yoon S.B., L e e K.S., K o o D.B., L e e D.S., Choo Y.K., Huh J.W., L e e S.R., K i m S.U., K i m S.H., K i m H.M., Chang K.T. (2001). Inactivated Sendai-virusmediated fusion improves early development of cloned bovine embryos by avoiding endoplasmicreticulum-stress-associated apoptosis. Reprod. Fert. Develop., 23: 826-836.

Song B.S., Yoon S.B., K im J.S., S i m B.W., Kim Y.H., Cha J.J., Choi S.A., Min H.K., L e e Y., Huh J.W., L e e S.R., Kim S.H., K o o D.B., Ch o o Y.K., K im H.M., K im S.U., $\mathrm{Ch}$ a $\mathrm{g}$ K.T. (2012). Induction of autophagy promotes preattachment development of bovine embryos by reducing endoplasmic reticulum stress. Biol. Reprod., 87: 1-11.

Staunstrup N.H., Stenderup K., Mortensen S., Primo M.N., Rosada C., Steiniche T., Liu Y., Li R., S chmi d t M., Purup S., Dagnæs-Hansen F., S chrøder L.D., S ven s s on L., P et er s en T.K., Calle s en H., B olund L., Mik k el s en J.G. (2017). Psoriasiform skin disease in transgenic pigs with high-copy ectopic expression of human integrins $\alpha 2$ and $\beta 1$. Dis. Model. Mech., 10: 869-880.

Tanabe Y., Kuwayama H., Wakayama S., Nagatomo H., Ooga M., Kamimura S., K i s h ig a m i S., Wa k a y m a T. (2017). Production of cloned mice using oocytes derived from ICR-outbred strain. Reproduction, 154: 859-866.

Tanida I., U e no T., K o m in a mi E. (2004). LC3 conjugation system in mammalian autophagy. Int. J. Biochem. Cell Biol., 36: 2503-2518.

Ts u ka d a M., O h s u mi Y. (1993). Isolation and characterization of autophagy-defective mutants of Saccharomyces cerevisiae. FEBS Lett., 333: 169-174.

Ts u k a mot o S. (2014). Autophagic activity as an indicator for selecting good quality embryos. Reprod. Med. Biol., 14: 57-64.

Tsukamoto S., Yamamoto A. (2013). The role of autophagy in early mammalian embryonic development. J. Mamm. Ova. Res., 30: 86-94.

V a j t a G. (2007). Handmade cloning: the future way of nuclear transfer? Trends Biotechnol., 25: 250-253.

Verma G., Arora J.S., S ethi R.S., Mukhopadhy y C.S., Verma R. (2015). Handmade cloning: recent advances, potential and pitfalls. J. Anim. Sci. Biotechnol., 6: 43. 
Wa ka y a ma T., P erry A.C., Z u c c ott i M., J o hn s on K.R., Yan a g i ma ch i R. (1998). Fullterm development of mice from enucleated oocytes injected with cumulus cell nuclei. Nature, 394: 369-374.

Wang H., Cu i W., Meng C., Zhang J., Li Y., Q i an Y., Xing G., Zh a o D., C a o S. (2018). MC1568 enhances histone acetylation during oocyte meiosis and improves development of somatic cell nuclear transfer embryos in pig. Cell. Reprogram., 20: 55-65.

Wang M., Ga o Y., Q u P., Q ing S., Q i a o F., Z hang Y., Mage r J., Wang Y. (2017). Spermborne miR-449b influences cleavage, epigenetic reprogramming and apoptosis of SCNT embryos in bovine. Sci. Rep., 7: 13403.

Wa n i N.A., Vettic al B.S., Hong S.B. (2017). First cloned Bactrian camel (Camelus bactrianus) calf produced by interspecies somatic cell nuclear transfer: A step towards preserving the critically endangered wild Bactrian camels. PLoS One, 12 (5): e0177800.

Wi $1 \mathrm{~m}$ u t I., S c hn ie ke A.E., M c W h i r J., K in d A.J., C a m p be 11 K.H. (1997). Viable offspring derived from fetal and adult mammalian cells. Nature, 385: 810-813.

Wongsrike a o P., Nagai T., Agung B., Taniguchi M., Kun ishi M., Suto S., O toi T. (2007). Improvement of transgenic cloning efficiencies by culturing recipient oocytes and donor cells with antioxidant vitamins in cattle. Mol. Reprod. Dev., 74: 694-702.

X u Y.N., S h e n X.H., L e e S.E., K w o n J.S., K i m D.J., H e o Y.T., C u i X.S., K i m N.H. (2012). Autophagy influences maternal mRNA degradation and apoptosis in porcine parthenotes developing in vitro. J. Reprod. Dev., 58: 576-584.

Yu M., Q i u Z.L., L i H., Z en g W.S., Che n L.N., Li Q.H., Q u an S. (2011). Association between cell apoptosis and the quality of early mouse embryos. Nan Fang Yi Ke Da Xue Xue Bao, 31: 409-413.

Yue Z., Jin S., Yang C., L e vine A.J., He in t z N. (2003). Beclin 1, an autophagy gene essential for early embryonic development, is a haploinsufficient tumor suppressor. Proc. Natl. Acad. Sci. USA., 100: 15077-15082.

Zakhartchenko V., Mueller S., A lberio R., Schernthaner W., Stojkovic M., Wenigerkind H., Wanke R., Las sn ig C., Mueller M., Wolf E., B re m G. (2001). Nuclear transfer in cattle with non-transfected and transfected fetal or cloned transgenic fetal and postnatal fibroblasts. Mol. Reprod. Dev., 60: 362-369.

Zh ang L., Hu ang Y., Wu Y., S i J., H u ang Y., Ji ang Q., L a n G., Gu o Y., J i a ng H. (2017). Scriptaid upregulates expression of development-related genes, inhibits apoptosis, and improves the development of somatic cell nuclear transfer mini-pig embryos. Cell. Reprogram., 19: 19-26.

Zhang P., Li u P., D ou H., Chen L., Chen L., Lin L., Tan P., Vaj ta G., Ga o J., Du Y., M a R.Z. (2013). Handmade cloned transgenic sheep rich in omega-3 fatty acids. PLoS One, 8 (2): e55941.

Zhang Y., Qu P., Ma X., Qiao F., Ma Y., Qing S., Zhang Y., Wang Y., Cui W. (2018). Tauroursodeoxycholic acid (TUDCA) alleviates endoplasmic reticulum stress of nuclear donor cells under serum starvation. PLoS One, 13 (5): e0196785.

Received: 14 VI 2018

Accepted: 1 X 2018 